\title{
Heterodyne Random Bit Generation Using an Optically Injected Semiconductor Laser in Chaos
}

\author{
Xiao-Zhou Li and Sze-Chun Chan, Member, IEEE
}

\begin{abstract}
Heterodyne generation of parallel random bit streams from chaotic emission of an optically injected semiconductor laser is investigated. The continuous-wave optical injection invokes chaotic dynamics in the laser. The broadband chaotic emission is detected through optical heterodyning and electrical heterodyning into different channels. The channels digitize the signals into parallel independent random bit streams. Because of efficient utilization of different portions of the chaos bandwidth, heterodyne detections enable parallel generation of random bit streams, offer high total output bit rates, and require no highbandwidth analogue-to-digital converters. In the experiment, two optical heterodyne channels and four electrical heterodyne channels are implemented. Each channel is required to digitize only $2.5 \mathrm{GHz}$ of a much broader chaos bandwidth. The sampling rate is $10 \mathrm{GHz}$ with five least significant bits selected from every 8-bit sample. The total output bit rate reaches $100 \mathrm{~Gb} / \mathrm{s}$ and $200 \mathrm{~Gb} / \mathrm{s}$ for optical and electrical heterodyning, respectively. The standard test suite of the National Institute of Standards and Technology verifies the randomness of both individual and interleaved output bit streams.
\end{abstract}

Index Terms-Random bit generation, chaos, heterodyne detection, optical injection, semiconductor lasers.

\section{INTRODUCTION}

$\mathbf{R}$ ANDOM bit generation (RBG) is essential for a variety of applications such as Monte-Carlo computations, data encryptions, and key distributions in secure communications [1]-[4]. Physical RBGs based on physical entropy sources are often needed to ensure the performances of these applications [5]-[18], despite the availability of advanced algorithms for pseudo RBG [19], [20]. High output bit rates in RBG are also desirable for high-speed computations and secure key distributions [21], [22]. Since photonic devices can both provide physical entropy and support fast bit rates, they offer some of the best solutions to high-speed RBG based on quantum measurements, optical noise, and chaotic dynamics. Quantum-based approaches employ quantum measurements on photons, which include quantifying the photon arrival time, polarization fluctuations, and phase fluctuations [23]-[31]. These quantum measurements ensure randomness

Manuscript received June 17, 2013; revised August 13, 2013; accepted August 18, 2013. Date of publication August 21, 2013; date of current version September 5, 2013. This work was supported in part by a Grant from the City University of Hong Kong under Project 7002726 and in part by a Grant from the Research Grant Council of Hong Kong under Project CityU 111210.

X.-Z. Li is with the Department of Electronic Engineering, City University of Hong Kong, Hong Kong, China (e-mail: xiaozli4-c@ my.cityu.edu.hk).

S.-C. Chan is with the Department of Electronic Engineering and the State Key Laboratory of Millimeter Waves, City University of Hong Kong, Hong Kong, China (e-mail: scchan@ cityu.edu.hk).

Color versions of one or more of the figures in this paper are available online at http://ieeexplore.ieee.org.

Digital Object Identifier 10.1109/JQE.2013.2279261 of the output, although the bit rate is often limited by the speed of single-photon detectors to the order of $1 \mathrm{~Gb} / \mathrm{s}$ [23]. Noise-based approaches employ high-speed photodetectors to yield electrical fluctuations from optical noise sources such as superluminescent light-emitting diodes and optical amplifiers [4], [32]-[35]. The fluctuations can be processed to generate random bits at hundreds of gigabits per second, but the electrical bandwidths of the photodetectors typically allow processing less than $1 \%$ of the noise spectrum. Proper optical bandpass filtering was often required to avoid damaging the photodetectors [36]. Supercontinuum generation dynamics seeded by noise were also investigated for RBG [37].

Pioneered by Uchida et al. [1], chaos-based RBG using semiconductor lasers continues to attract much investigations [2]-[4], [7]-[18]. The chaotic dynamics typically produce intensity fluctuations with bandwidths matching those of high-speed photodetectors [38]-[42]. They give physical entropy by magnifying the effects of intrinsic laser noise through chaotic mixing [43]-[46]. The lasers can be synchronized when properly coupled [47], [48]. In previous investigations on RBG, chaotic dynamics were invoked by optical feedback of various forms including feedback from a mirror [1]-[3], [7]-[9], feedback incorporating injection for bandwidth broadening [10]-[13], feedback into a ring laser through a fiber loop [14], feedback within two-section or mutually coupled lasers [15], [16], and feedback with polarization rotation for high robustness [17]. Typically, the chaotic laser emission is converted by a photodetector into an electrical signal. The signal is then sampled and digitized by an analogue-to-digital converter (ADC) into raw sample bits. These raw bits are subsequently transformed into the output bits though different postprocessing operations, including delayed exclusive-OR (XOR), high-order differentiations, and least significant bits (LSBs) selection. As examples, chaosbased RBG has been investigated at $1.7 \mathrm{~Gb} / \mathrm{s}$ by using ADCs of 1-bit resolution [1], $75 \mathrm{~Gb} / \mathrm{s}$ by selecting $6 \mathrm{LSBs}$ per 8-bit sample [10], $140 \mathrm{~Gb} / \mathrm{s}$ by extracting the internal noiseenhanced 16-bit samples in ADCs [8], $300 \mathrm{~Gb} / \mathrm{s}$ by deducing 15 bits from 8-bit raw samples through a high-order differentiation [3], and $400 \mathrm{~Gb} / \mathrm{s}$ by incorporating bit-orderreversal to scramble the spectrum of the raw samples [4], [13], which also resulted in a bit rate of $1.05 \mathrm{~Tb} / \mathrm{s}$ by using four lasers concurrently [49]. However, as the previous experiments adopted optical feedback to invoke chaos, the chaotic emissions were often associated with time-delay signatures such that residual autocorrelation at the feedback round-trip time imposed limitations on the sampling rate [17], [50]-[52]. Moreover, a high-bandwidth ADC is typically required for 


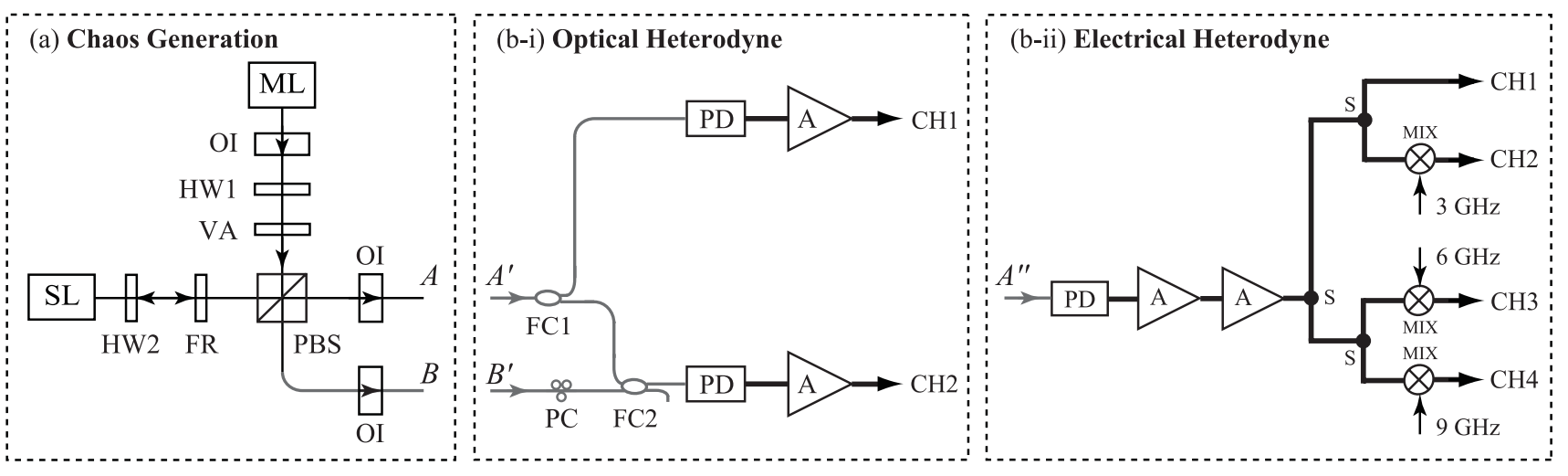

Fig. 1. Experimental setup for parallel RBG from a chaotic laser. (a) Chaos generation using optical injection. (b-i) Optical heterodyne detection of chaos into two channels by connecting points $A$ to $A^{\prime}$ and $B$ to $B^{\prime}$. (b-ii) Electrical heterodyne detection of chaos into four channels by connecting point $A$ to $A^{\prime \prime}$. ML: master laser. SL: slave laser. OI: optical isolator. HW: half-wave plate. VA: variable attenuator. PBS: polarizing beam splitter. FR: Faraday rotator. FC: fiber coupler. PC: polarization control. PD: photodetector. A: microwave amplifier. S: microwave power splitter. MIX: microwave mixer. Gray lines: optical fibers. Thin lines: free-space optical paths. Thick lines: microwave cables. Signal in each channel is subsequently processed into a stream of random bits.

capturing the broadband chaos spectrum. The ADC bandwidth usually exceeded at least half the sampling rate to realize undersampling, which violated the Nyquist criterion deliberately to produce uncorrelated output bits [9], [10]. Furthermore, for high-speed RBG over $100 \mathrm{~Gb} / \mathrm{s}$, digital postprocessing was often responsible for boosting the output bit rate [3], [4], [8], [13], [49]. Output bit rates faster than or equal to the raw bit rates had been investigated even when the raw bits are not totally random.

In this paper, parallel RBG through heterodyne detection of chaotic emission from an optically injected semiconductor laser is investigated. Instead of optical feedback, the continuous-wave $(\mathrm{CW})$ optical injection invokes chaotic dynamics which are free from any time-delay signatures. By optical and electrical heterodyne detections, the chaotic emission is transformed into parallel channels of electrical signals. The signal in each channel is then digitized into independent random bit streams. As a result, different portions of the chaos spectrum are efficiently utilized by the heterodyne detections to offer the following advantages. Firstly, parallel random bit streams can be generated from just one laser in chaos because different spectral ranges are utilized. Secondly, low-bandwidth ADCs can be adopted because each channel processes only a small portion of the chaos bandwidth. Thirdly, by collecting the bits from all channels, high total output bit rates exceeding $100 \mathrm{~Gb} / \mathrm{s}$ can be achieved, which requires no digital postprocessing that boosts the output bit rate above the raw bit rate.

Experimentally, two optical heterodyne channels and four electrical heterodyne channels are implemented. Each channel uses a low-bandwidth ADC to digitize only $2.5 \mathrm{GHz}$ of a much broader chaos spectrum. At a sampling rate of $10 \mathrm{GHz}$, 5 LSBs are selected from every 8-bit sample so that the total rate of RBG reaches $100 \mathrm{~Gb} / \mathrm{s}$ and $200 \mathrm{~Gb} / \mathrm{s}$ for optical and electrical heterodyning, respectively. Although the channels are derived from a single chaos spectrum, the randomness of the output bits is verified for all channels individually and collectively, according to the standard test suite of the National Institute of Standards and Technology (NIST). In a

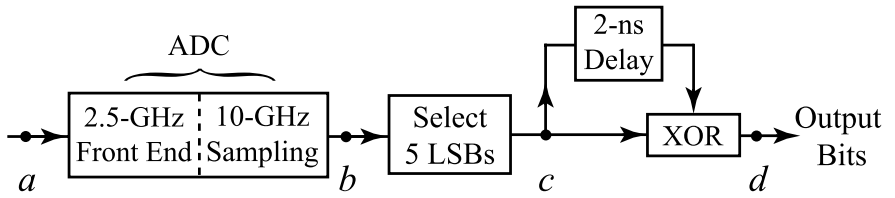

Fig. 2. Digitization and postprocessing in each channel for RBG.

recent work, the optically injected semiconductor laser was investigated for 40-Gb/s RBG using the low-bandwidth ADC with LSBs selection [18], [53]. The low-bandwidth ADC was sufficient because of oversampling at a sampling rate of $10 \mathrm{GHz}$, which is above the Nyquist rate. Randomness was ensured due to the spectral broadening associated with LSBs selection [18], [53]. Nonetheless, the approach utilized only a small fraction of the chaos bandwidth. To further increase the bit rate, utilization of different portions of the chaos bandwidth is necessary.

Following this introduction, the experimental setup for heterodyne parallel RBG is illustrated in Section II. The experimental results are described in detail in Section III, which is followed by a discussion and a conclusion in Sections IV and V, respectively.

\section{EXPERIMENTAL SETUP}

The schematic of the experimental setup is shown in Figs. 1 and 2. There are three parts in the setup: chaos generation, heterodyne detection, and digital processing, which correspond to Figs. 1(a), 1(b), and 2, respectively.

\section{A. Chaos Generation}

Figure 1(a) shows the generation of chaos by perturbing a semiconductor laser using CW optical injection. The semiconductor lasers used are single-mode distributed-feedback lasers (Mitsubishi ML920T43S-01) arranged in a master-slave configuration. The slave laser is biased above threshold at $40 \mathrm{~mA}$ and temperature stabilized at $26.5^{\circ} \mathrm{C}$. When there is no optical injection, the slave laser emits at its freerunning wavelength of $1548.42 \mathrm{~nm}$ with an output power of 


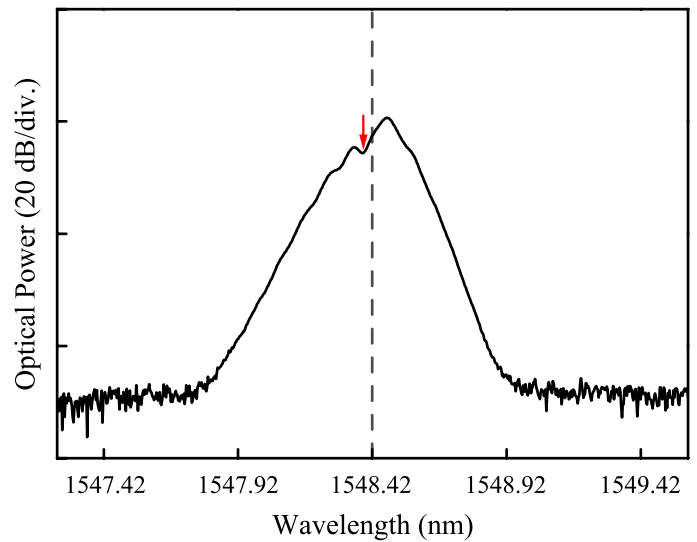

Fig. 3. Optical spectrum of the chaotic emission from the slave laser. The arrow marks the wavelength of the injection light. The dashed line marks the free-running wavelength of the slave laser. Resolution bandwidth: $0.06 \mathrm{~nm}$.

$10 \mathrm{~mW}$. Its relaxation resonance frequency is about $11 \mathrm{GHz}$. The master laser is biased above threshold at $128.5 \mathrm{~mA}$ and temperature stabilized at $18.6^{\circ} \mathrm{C}$. The optical frequency detuning of the master laser from the free-running slave laser is about $4 \mathrm{GHz}$, which corresponds to a wavelength detuning of roughly $-0.03 \mathrm{~nm}$.

The CW emission of the master laser passes through an optical isolator that prevents optical feedback from backreflections. The linearly polarized emission then goes through halfwave plate HW1, variable attenuator VA, and polarizing beam splitter PBS which splits the emission into two beams. The total power and the power ratio of the two beams are controlled by VA and HW1, respectively. The transmitted beam from PBS is coupled directly into an optical fiber. Thus, CW emission of the master laser can be collected at point $B$ after an isolator. The reflected beam from PBS is injected into the slave laser through Faraday rotator FR and half-wave plate HW2, which match the polarization of the injection to that of the slave laser. The combination of PBS, FR, and HW2 acts as a free-space circulator. It realizes unidirectional injection from the master laser to the slave laser with about $0.3 \mathrm{~mW}$ impinging onto the slave laser facet, while the slave laser emission is transmitted through PBS and an isolator to point $A$.

The optical spectrum of the slave laser emission collected at point $A$ is shown in Fig. 3, which is obtained from an optical spectrum analyzer (Agilent 86140B) with 0.06-nm resolution bandwidth. The broadband optical spectrum is attributed to the chaotic dynamics of the slave laser invoked by the CW optical injection [40]-[42], [54]-[57]. As commonly observed in optically injected lasers, the spectral peak of the optical chaos spectrum is shifted away from the free-running wavelength of the slave laser (as marked by the dashed line). Also, the regeneration of the injected light contributes to less than $5 \%$ of the optical power so there is no apparent peak at the injection wavelength (as marked by the arrow).

\section{B. Heterodyne Detection}

The chaotic emission from the slave laser of Fig. 1(a) can be converted into multichannel electrical signals by incorporating optical heterodyne detection and electrical heterodyne detections as shown in Fig. 1(b-i) and (b-ii), respectively. Two parallel channels of electrical signals are obtained using optical heterodyne detection, while four parallel channels of electrical signals are obtained using electrical heterodyne detection.

For optical heterodyne detection, points $A$ and $B$ of Fig. 1(a) are connected to points $A^{\prime}$ and $B^{\prime}$ in Fig. 1(b-i) accordingly. The chaotic emission from the slave laser is split by a fiber coupler FC1 in Fig. 1(b-i). One output arm of FC1 sends $0.5 \mathrm{~mW}$ of the chaotic emission directly to a photodetector (Newport AD-10ir) followed by a microwave amplifier (HP 83006A) in forming channel CH1. The other output arm of FC1 sends $0.15 \mathrm{~mW}$ of the chaotic emission to a 50:50 fiber coupler FC2, which combines the chaotic emission with $1 \mathrm{~mW}$ of $\mathrm{CW}$ emission from the master laser. An output from FC2 is then connected to a photodetector and a microwave amplifier in forming channel $\mathrm{CH} 2$. Therefore, $\mathrm{CH} 2$ implements optical heterodyne detection of the chaotic emission from the slave laser using the master laser as the optical local oscillator. The beat signal amplitude is maximized by using a polarization control immediately after point $B^{\prime}$ for matching the polarizations of the master laser emission and the chaotic emission at FC2. The optical power delivered from FC2 to the photodetector is set at the largest value as limited by its damage threshold. Maximization of the amplitude of the beat signal requires setting the optical power from the chaotic emission equal to that from the $\mathrm{CW}$ emission. However, due to direct detection of the chaotic emission in $\mathrm{CH} 2, \mathrm{CH} 2$ also contains a signal component that is the same as the signal in $\mathrm{CH} 1$. In order to reduce the undesirable cross-correlation between the two channels, the optical power of the chaotic emission is set smaller than that of the $\mathrm{CW}$ emission at the photodetector in the experiment.

For electrical heterodyne detection, point $A$ of Fig. 1(a) is connected to point $A^{\prime \prime}$ in Fig. 1(b-ii). About $1 \mathrm{~mW}$ of the chaotic emission from the slave laser is detected by a photodetector followed by two microwave amplifiers. The electrical output is then split equally, through the microwave power splitters, into four channels as shown in Fig. 1(b-ii). Other than channel $\mathrm{CH} 1$, each channel adopts electrical heterodyne by using a microwave mixer driven by a microwave local oscillator. The oscillators generate sinusoidal signals at $3 \mathrm{GHz}, 6 \mathrm{GHz}$, and $9 \mathrm{GHz}$ for channels $\mathrm{CH} 2, \mathrm{CH} 3$, and $\mathrm{CH} 4$, respectively.

In Fig. 1(b), each amplifier has a gain of $20 \mathrm{~dB}$ with a bandwidth of $26.5 \mathrm{GHz}$ and each photodetector has a responsivity of about $150 \mathrm{~V} / \mathrm{W}$ with a bandwidth of $43 \mathrm{GHz}$. In practice, the large electronic bandwidths are not needed because of the much lower ADC front-end bandwidth adopted in the following digital processing.

\section{Digital Processing}

For each channel in Fig. 1(b), the electrical output signal is sent to an ADC in a real-time oscilloscope (Agilent 90254A) incorporating digital signal processing. The steps of digitization and postprocessing are shown in Fig. 2. At position $a$, the electrical signal is first low-pass filtered by the ADC front-end that has a low bandwidth of $2.5 \mathrm{GHz}$. 
It also blocks frequency components below $10 \mathrm{MHz}$ and has a stopband attenuation of $100 \mathrm{~dB}$. The ADC digitizes the signal at a sampling rate of $10 \mathrm{GHz}$. This is regarded as oversampling because the sampling rate is greater than twice the front-end bandwidth. The ADC has a 8-bit resolution so that the raw bit rate per channel is $80 \mathrm{~Gb} / \mathrm{s}$ at position $b$. In the subsequent postprocessing, only 5 LSBs are selected for every raw sample in order to ensure randomness [14], [18]. Each sample at position $c$ is then compared with its 2-ns delayed replica through a bitwise XOR operation. The XOR operation is commonly adopted to reduce any bias of bits [16], [52], where the choice of the delay time is not critical as long as it is sufficiently large to avoid correlation between the original and the delayed samples [10]. An output random bit stream is generated at position $d$ at an output bit rate of $50 \mathrm{~Gb} / \mathrm{s}$ per channel. Since the oscilloscope has four ADCs, it supports parallel processing of at most four channels. The total output rate of RBG is $100 \mathrm{~Gb} / \mathrm{s}$ and $200 \mathrm{~Gb} / \mathrm{s}$ using the two and four channels from optical and electrical heterodyne detections, respectively.

\section{EXPERIMENTAL RESULTS}

The experimental results of RBG using optical heterodyne detection in Fig. 1(b-i) and electrical heterodyne detection in Fig. 1(b-ii) are investigated in this section. Different power spectra obtained from different heterodyne detection channels are first examined. Their corresponding time series, autocorrelations, and cross-correlations are then presented, which elucidate the independence among the channels. The dependencies of randomness on the output bit rates through adjusting the number of LSBs selected are also examined. For randomness verifications, the NIST Special Publication 800-22 statistical tests are employed [58], which collect the statistics of 10001 -Mbit sequences. At significance level $\alpha=0.01$, the success proportion should be in the range of $0.99 \pm 0.0094392$. The composite $P$-value should be larger than 0.0001 for uniformity.

\section{A. Optical Heterodyne RBG}

By optical heterodyne detection, chaotic emission from the slave laser in Fig. 1(a) results in channels $\mathrm{CH} 1$ and $\mathrm{CH} 2$ in Fig. 1(b-i). The electrical signal in each channel is further processed through the steps in Fig. 2. Figure 4(a) shows the power spectra of $\mathrm{CH} 1$ and $\mathrm{CH} 2$, which are measured at position $a$ of Fig. 2 using a power spectrum analyzer (Agilent N9010A). The power spectrum of CH1 in Fig. 4(a) is from direct detection of the chaotic emission. Only the optical field amplitude, not the optical phase, of the chaotic emission contributes to $\mathrm{CH} 1$. The spectrum has a broad effective bandwidth of $7.5 \mathrm{GHz}$ according to the convention of $80 \%$ total power containment [59]. It peaks at around the relaxation resonance frequency of the slave laser, which is a common feature for chaotic semiconductor lasers [18], [40], [60]. The power spectrum of $\mathrm{CH} 2$ in Fig. 4(a) is from optical heterodyne detection of the chaotic emission with the master laser acting as the local oscillator. Both the optical field amplitude and the optical phase of the chaotic emission contribute to $\mathrm{CH} 2$.

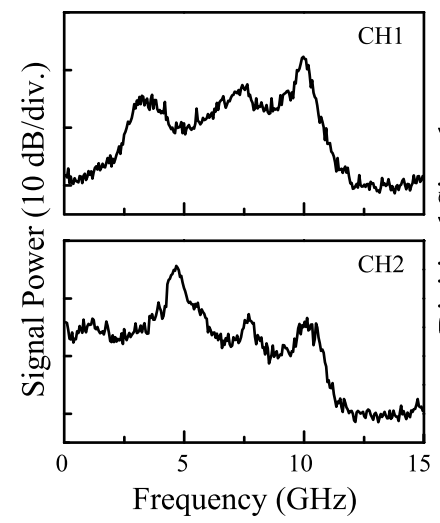

(a)

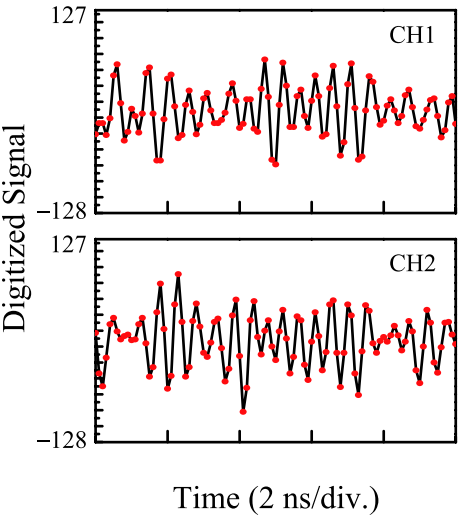

(b)
Fig. 4. (a) Power spectra and (b) time series from channels $\mathrm{CH} 1$ and $\mathrm{CH} 2$ of optical heterodyne detection. The dots in the time series are the digitized samples.

The spectrum is mainly a result of downshifting the chaotic optical spectrum by the optical frequency of the master laser, which is caused by beating between the chaotic emission of the slave laser and the much stronger $\mathrm{CW}$ emission of the master laser at the photodetector. Although the chaotic emission also contains a regenerative component at the optical frequency of the master laser, it is very weak compared to the total power of the chaotic emission [56], [57]. As a result, the power spectrum of $\mathrm{CH} 2$ is significantly different from that of $\mathrm{CH} 1$.

Figure 4(b) shows the time series of $\mathrm{CH} 1$ and $\mathrm{CH} 2$ that are simultaneously measured by the oscilloscope and recorded at position $b$ of Fig. 2. For each channel, the voltage detection window size of the ADC is set at $4.5 \sigma$, where $\sigma$ is the standard deviation of the voltage distribution in the time series [4], [35]. Thus, voltage values within $\pm 4.5 \sigma$ are evenly divided into 256 digitization levels of the 8-bit ADC. The time series in Fig. 4(b) correspond to the power spectra in Fig. 4(a) after being low-pass filtered by the $2.5-\mathrm{GHz}$ front ends of the ADCs. The data points sampled at $10 \mathrm{GHz}$ are shown as the dots in Fig. 4(b). The time series appear irregular due to the chaotic dynamics, but it is necessary to examine the autocorrelations and cross-correlation of the channels in Figs. 5 and 6. The correlation curves are calculated using $10^{6}$ samples for each channel.

In Fig. 5(a), the autocorrelation curves of the 8-bit digitized signals in $\mathrm{CH} 1$ and $\mathrm{CH} 2$ are plotted in black and blue, respectively. Each autocorrelation curve contains no peaks other than the main peak at zero delay. This is because chaos is generated by optical injection instead of feedback. The chaotic dynamics contain no feedback round-trip time and thus produce no time-delay signatures in the autocorrelation [50], [51], [61]. However, due to oversampling, the main peak of the autocorrelation curve has an observable width. The magnitudes of autocorrelations drop to below $50 \%$ only when the delay time is greater than $0.5 \mathrm{~ns}$, which corresponds to a delay of 5 samples. Nonetheless, for every sample, only 5 LSBs are selected and processed after position $c$ in Fig. 2. Such selection of LSBs is a common procedure for improving the uniformity of bit distributions [10], [17], [53]. It is also a nonlinear operation that effectively broadens the spectra of 


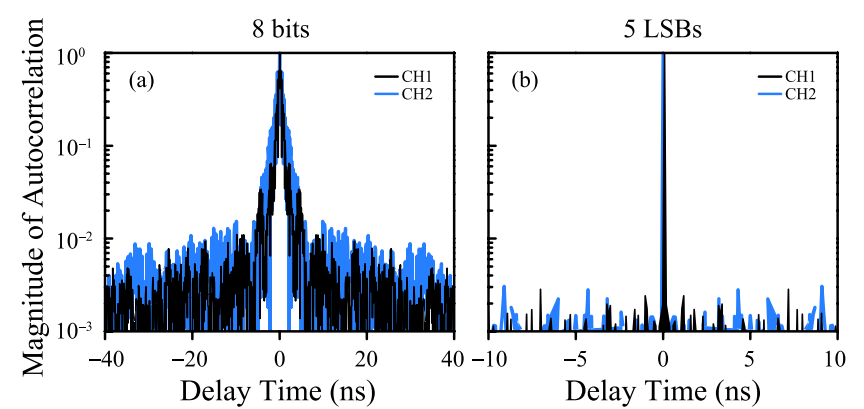

Fig. 5. Autocorrelations of digitized signals from $\mathrm{CH} 1$ and $\mathrm{CH} 2$ in optical heterodyne detection. (a) All 8 bits and (b) only 5 LSBs are used per sample.

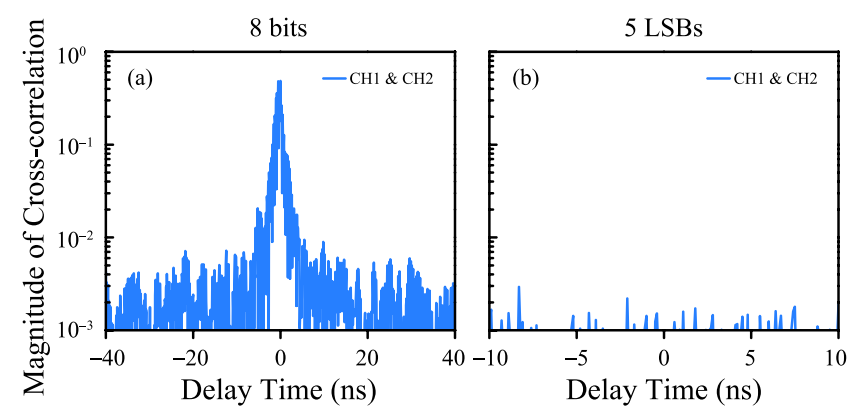

Fig. 6. Cross-correlation between the digitized signals of $\mathrm{CH} 1$ and $\mathrm{CH} 2$ in optical heterodyne detection. (a) All 8 bits and (b) only 5 LSBs are used per sample.

the digitized signals [14], [18], [26]. So the autocorrelation curves for the digitized signals using only the 5 LSBs are significantly narrowed, as Fig. 5(b) shows. The magnitudes of autocorrelations are less than $0.4 \%$ as long as the delay time is at least $0.1 \mathrm{~ns}$, which requires a delay of just 1 sample.

In Fig. 6(a), the cross-correlation curve between the signals in $\mathrm{CH} 1$ and $\mathrm{CH} 2$ is plotted using all 8 bits per sample. A residual cross-correlation peak of $48.6 \%$ is observed in Fig. 6(a). This is because, according to Fig. 1(b-i), CH2 partially contains a signal component from direct detection of the chaotic emission. The component is independent of the $\mathrm{CW}$ emission of the master laser and is the same as the signal in $\mathrm{CH} 1$. Nonetheless, as a heterodyne detection channel, $\mathrm{CH} 2$ contains a much stronger beat signal component between the master laser emission and the chaotic emission, which ensures that $\mathrm{CH} 1$ and $\mathrm{CH} 2$ are not highly correlated. Moreover, as Fig. 6(b) shows, the cross-correlation can be completely eliminated when only 5 LSBs are selected per sample. This is because the LSBs correspond to the fine details of the time series. The magnitude of cross-correlation is always less than $0.4 \%$. In short, with the selection of LSBs in Figs. 5(b) and 6(b), the digitized signals are not correlated either within individual channels or across different channels.

The output bits for each channel are produced at position $d$ in Fig. 2, where every sample is compared with its delayed replica by the XOR operation to suppress any bias of bits [18], [52]. Each individual channel has an output bit rate of $50 \mathrm{~Gb} / \mathrm{s}$, while $\mathrm{CH} 1$ and $\mathrm{CH} 2$ collectively gives a total output bit rate of $100 \mathrm{~Gb} / \mathrm{s}$. To verify the mutual independence between the two channels, their output bits are interleaved by
TABLE I

Nist Test Results on Optical Heterodyne RBG at $100 \mathrm{~Gb} / \mathrm{s}$

\begin{tabular}{lccc}
\hline Statistical test & $P$-value & Proportion & Result \\
\hline Frequency & 0.030399 & 0.9940 & Success \\
Block-frequency & 0.639202 & 0.9880 & Success \\
Cumulative-sums & 0.119508 & 0.9920 & Success \\
Runs & 0.178604 & 0.9880 & Success \\
Longest-run & 0.858002 & 0.9960 & Success \\
Rank & 0.024356 & 0.9920 & Success \\
FFT & 0.439122 & 0.9890 & Success \\
Nonoverlapping-templates & 0.003346 & 0.9810 & Success \\
Overlapping-templates & 0.973055 & 0.9920 & Success \\
Universal & 0.392456 & 0.9860 & Success \\
Approximate-entropy & 0.189625 & 0.9850 & Success \\
Random-excursions & 0.026042 & 0.9838 & Success \\
Random-excursions-variant & 0.015903 & 0.9822 & Success \\
Serial & 0.814724 & 0.9870 & Success \\
Linear-complexity & 0.002447 & 0.9890 & Success \\
\hline Total & & & 15 \\
\hline
\end{tabular}

taking one bit from $\mathrm{CH} 1$ and $\mathrm{CH} 2$ alternatively in forming an interleaved output bit stream for randomness testing [62]. Table I shows the successful NIST test results of the interleaved bits. Therefore, with simply LSBs selection and XOR processing, optical heterodyne detection yields parallel RBG at $100-\mathrm{Gb} / \mathrm{s}$.

The number of passes in the NIST statistical tests is then examined as the digitization settings are varied in Figs. 7 and 8. The output bits are considered random if they pass all 15 tests. In Fig. 7, the gray curves show the results for the individual channels $\mathrm{CH} 1$ and $\mathrm{CH} 2$ as the detection window size of the ADCs is varied. The detection window size is measured with respect to the standard deviation voltage $\sigma$ of the corresponding channel. At window size of $2.0 \sigma$, the input signals to the ADCs often exceed the detection windows. So the digitized signals are at their extreme values too frequently, which lead to failing some NIST tests. As the detection window size increases, the extreme values appear less frequently, so the number of passes increases to 15 . Such a dependence of the NIST results on the detection window size is commonly observed in some related work [4], [35]. For the interleaved output bits, the number of passes also increases to 15 when the detection window size increases, as the black curve in Fig. 7 shows. The detection window size is then fixed at $4.5 \sigma$ while the number of LSBs selected per sample is varied in Fig. 8. Varying the number of LSBs corresponds to varying the output bit rate because the sampling rate is fixed at $10 \mathrm{GHz}$. The gray curves in Fig. 8 show the NIST results for the individual channels $\mathrm{CH} 1$ and $\mathrm{CH} 2$. Each individual channel passes all 15 tests for up to 5 LSBs selected per sample, which gives the output bit rate of $50 \mathrm{~Gb} / \mathrm{s}$. The black curve shows the NIST results for the interleaved output bits. They also pass all 15 tests when up to 5 LSBs are selected per sample, where the two channels together generate bits at $100 \mathrm{~Gb} / \mathrm{s}$. For comparison, the chaotic emission from the slave laser is blocked by breaking the connection between $A$ and $A^{\prime}$ in Fig. 1. The outputs due to electronic noise fail the NIST tests unless just 1 LSB is selected per sample. Therefore, the chaotic emission is necessary for providing sufficiently 


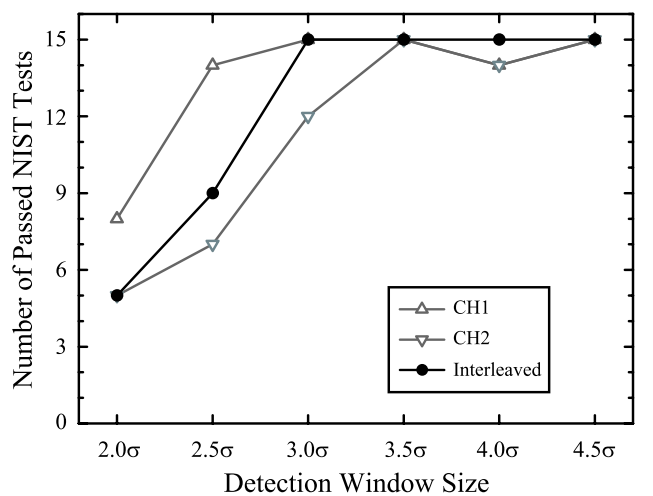

Fig. 7. Number of NIST tests passed versus the detection window size of the ADCs for optical heterodyne RBG. The NIST tests are conducted on the $50-\mathrm{Gb} / \mathrm{s}$ output bits from $\mathrm{CH} 1$ and $\mathrm{CH} 2$ separately. The sampling rate is $10 \mathrm{GHz}$ with $5 \mathrm{LSBs}$ selected per sample. The tests are also conducted on the interleaved output bits from the two channels with a total output rate of $100 \mathrm{~Gb} / \mathrm{s}$.

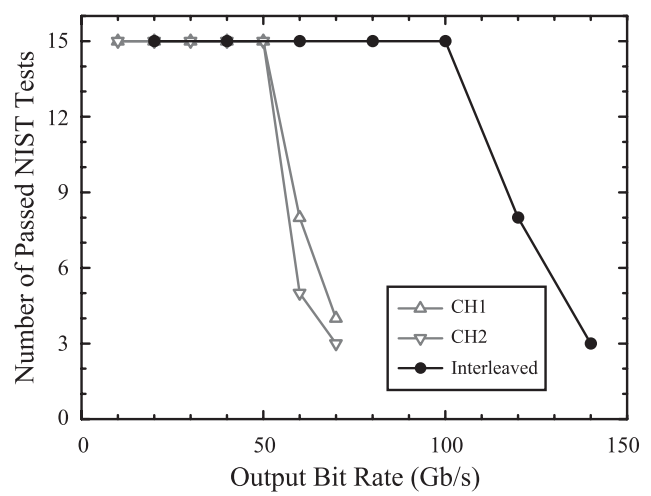

Fig. 8. Number of NIST tests passed versus the output bit rate for optical heterodyne RBG. The output bit rate is varied by adjusting the number of LSBs selected per sample. The sampling rate is fixed at $10 \mathrm{GHz}$. The detection window size of the ADCs is kept at $4.5 \sigma$. The NIST tests are conducted on $\mathrm{CH} 1, \mathrm{CH} 2$, and their interleaved output bits.

strong signals for high-speed RBG. The maximal total output bit rate of $100 \mathrm{~Gb} / \mathrm{s}$ is obtained from the optical heterodyne experiment using simple processing and low-bandwidth ADCs in the two channels.

\section{B. Electrical Heterodyne RBG}

By electrical heterodyne detection, chaotic emission from the slave laser in Fig. 1(a) results in channels $\mathrm{CH} 1$ to $\mathrm{CH} 4$ in Fig. 1(b-ii). Only the optical field amplitude, not the optical phase, of the chaotic emission is converted into electrical signals by the photodetector in contributing to the four channels. The output from each channel of Fig. 1(b-ii) follows the processing in Fig. 2, where the power spectrum at position $a$ is shown in Fig. 9(a). For CH1, the power spectrum is from direct detection of the chaotic emission. The broadband spectrum is similar to that for $\mathrm{CH} 1$ in Fig. 4(a), although there are slight differences due to the frequency responses of the microwave amplifiers and the microwave power splitters in Fig. 1(b-ii). The spectral peak at $10.25 \mathrm{GHz}$ in Fig. 9(a) is again close to the relaxation resonance frequency of the slave laser. However, only the spectrum below $2.5 \mathrm{GHz}$ is subsequently digitized due to low-pass filtering by the ADC front-end. For the other three

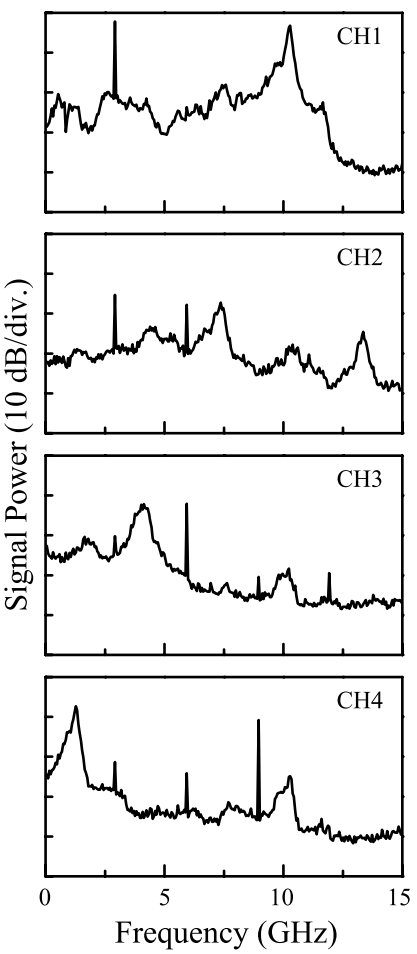

(a)
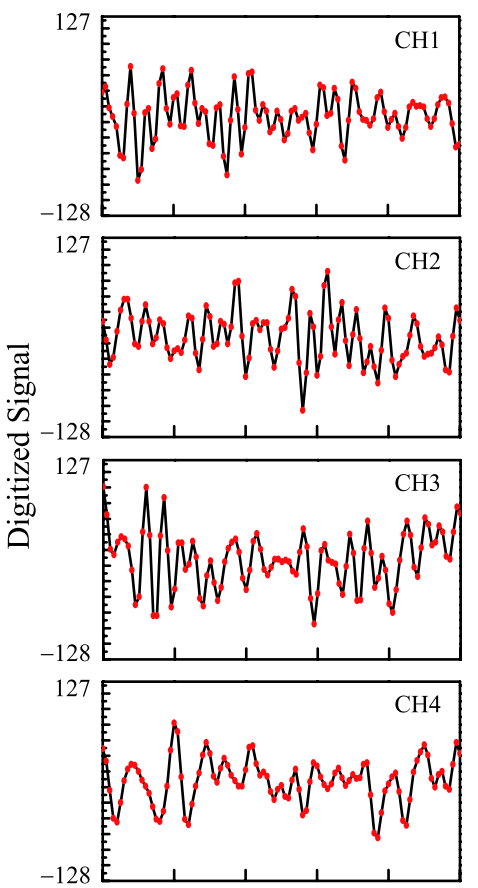

Time (2 ns/div.)

(b)
Fig. 9. (a) Power spectra and (b) time series from channels $\mathrm{CH} 1, \mathrm{CH} 2, \mathrm{CH} 3$, and $\mathrm{CH} 4$ of electrical heterodyne detection. The dots in the time series are the digitized samples.

channels, electrical heterodyne detection results in their power spectra in Fig. 9(a). The original power spectrum of $\mathrm{CH} 1$ is shifted by the local oscillator frequency in each channel, which explains the shift of the spectral peak to $7.25 \mathrm{GHz}$ in $\mathrm{CH} 2$, $4.25 \mathrm{GHz}$ in $\mathrm{CH} 3$, and $1.25 \mathrm{GHz}$ in $\mathrm{CH} 4$, respectively. The ADCs then process the signals below $2.5 \mathrm{GHz}$ from $\mathrm{CH} 2$, $\mathrm{CH} 3$, and $\mathrm{CH} 4$, which are respectively converted from the signals in $3 \pm 2.5 \mathrm{GHz}, 6 \pm 2.5 \mathrm{GHz}$, and $9 \pm 2.5 \mathrm{GHz}$ of the original power spectrum in $\mathrm{CH} 1$. Thus, electrical heterodyne detection allows processing different spectral regions of the original spectrum at frequencies above the bandwidths of the ADCs. It is also observed that, due to imperfect signal isolations in the mixers, there are narrow tones at the local oscillator frequencies in the spectra of Fig. 9(a). For instance, there is a leakage of the signal from the local oscillator at $3 \mathrm{GHz}$ through the mixer of $\mathrm{CH} 2$. It enters $\mathrm{CH} 1$ through the microwave power splitter in Fig. 1(b-ii) and appears as the sharp tone at $3 \mathrm{GHz}$ in the power spectrum of $\mathrm{CH} 1$ in Fig. 9(a). In order to prevent the leaked signals of the local oscillators from entering the ADCs in affecting the subsequent digital processing, the local oscillator frequencies greater than the bandwidths of the ADCs are chosen.

Figure 9(b) shows the time series measured simultaneously at position $b$ of Fig. 2 for the four channels. The time series are irregular due to the chaotic dynamics. For each channel, the detection window size of the ADC is set at $4.5 \sigma$ with $\sigma$ being the standard deviation of the voltage distribution. The dots in Fig. 9(b) are the data points sampled at $10 \mathrm{GHz}$. The correlation curves are examined in Figs. 10-12.

In Fig. 10(a), the autocorrelation curves of the 8-bit digitized signals in $\mathrm{CH} 1, \mathrm{CH} 2, \mathrm{CH} 3$, and $\mathrm{CH} 4$ are shown. The use of 


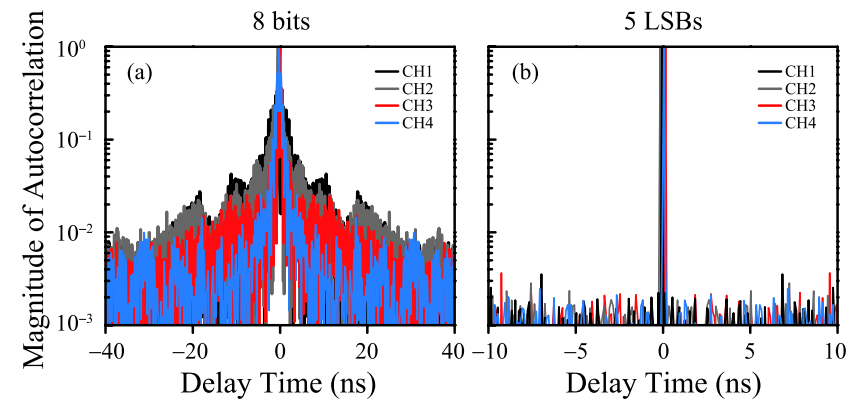

Fig. 10. Autocorrelations of digitized signals from $\mathrm{CH} 1, \mathrm{CH} 2, \mathrm{CH} 3$, and CH4 in electrical heterodyne detection. (a) All 8 bits and (b) only 5 LSBs are used per sample.

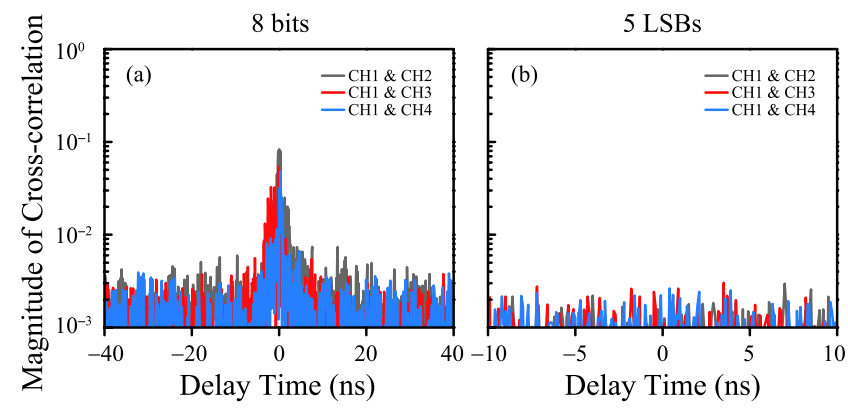

Fig. 11. Cross-correlations between the digitized signals of $\mathrm{CH} 1$ and the other three channels in electrical heterodyne detection. (a) All 8 bits and (b) only 5 LSBs are used per sample.

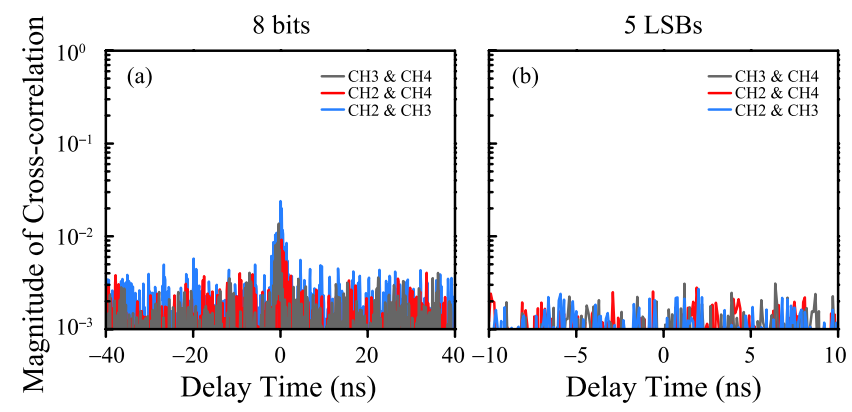

Fig. 12. Cross-correlations between the digitized signals of $\mathrm{CH} 2, \mathrm{CH} 3$, and $\mathrm{CH} 4$ in electrical heterodyne detection. (a) All 8 bits and (b) only 5 LSBs are used per sample.

optical injection for chaos generation again yields the autocorrelation curves with no strong peaks other than the main peaks at zero delay. Because of oversampling, the magnitudes of the main peaks drop to below $50 \%$ only when the delay time is greater than $0.4 \mathrm{~ns}$, which corresponds to 4 sampling periods. After position $c$ in Fig. 2, only 5 LSBs are selected per sample such that the autocorrelation curves are significantly narrowed, as Fig. 10(b) shows. The magnitudes of autocorrelations are always less than $0.6 \%$ as long as the delay time is at least $0.1 \mathrm{~ns}$, which requires a delay of just 1 sample.

In Fig. 11(a), the cross-correlations between the signal in $\mathrm{CH} 1$ and the signals in the rest of the channels are plotted using all 8 bits per sample. The curves show that $\mathrm{CH} 1$ is not much correlated with the other channels. The curves have peaks at zero delay because the mixers do not provide
TABLE II

NIST Test Results on Electrical Heterodyne RBG at $200 \mathrm{~Gb} / \mathrm{s}$

\begin{tabular}{lccc}
\hline Statistical test & $P$-value & Proportion & Result \\
\hline Frequency & 0.961039 & 0.9920 & Success \\
Block-frequency & 0.370262 & 0.9840 & Success \\
Cumulative-sums & 0.229559 & 0.9910 & Success \\
Runs & 0.118120 & 0.9920 & Success \\
Longest-run & 0.866097 & 0.9860 & Success \\
Rank & 0.429923 & 0.9870 & Success \\
FFT & 0.003297 & 0.9890 & Success \\
Nonoverlapping-templates & 0.004055 & 0.9820 & Success \\
Overlapping-templates & 0.605916 & 0.9890 & Success \\
Universal & 0.258307 & 0.9840 & Success \\
Approximate-entropy & 0.155499 & 0.9920 & Success \\
Random-excursions & 0.127762 & 0.9868 & Success \\
Random-excursions-variant & 0.019744 & 0.9835 & Success \\
Serial & 0.217857 & 0.9870 & Success \\
Linear-complexity & 0.595549 & 0.9920 & Success \\
\hline Total & & & 15 \\
\hline
\end{tabular}

perfect isolation, but the correlations are less than $8.3 \%$ in magnitude. Moreover, Fig. 12(a) shows the cross-correlations among the signals in $\mathrm{CH} 2, \mathrm{CH} 3$, and $\mathrm{CH} 4$ using all 8 bits per sample, where the residual correlations are less than $2.4 \%$ in magnitude. According to Figs. 11(b) and 12(b), the selection of 5 LSBs per sample successfully eliminates the cross-correlations between any two of the four channels to less than $0.5 \%$ in magnitude. Therefore, similar to optical heterodyne detection, electrical heterodyne detection can generate digitized signals which are not correlated within or across different channels.

After the XOR operation with the delayed samples with 5 LSBs, the output bits for each channel are produced at position $d$ in Fig. 2. Each individual channel has an output bit rate of $50 \mathrm{~Gb} / \mathrm{s}$, which means $\mathrm{CH} 1$ to $\mathrm{CH} 4$ collectively gives a total bit rate of $200 \mathrm{~Gb} / \mathrm{s}$. To verify the mutual independence among the four channels, their output bits are interleaved in the order of $\mathrm{CH} 1$ to $\mathrm{CH} 4$, where one bit is taken from a channel at a time. The interleaved bits pass all of the 15 NIST tests as Table II shows. Therefore, randomness is verified for 200-Gb/s parallel RBG using electrical heterodyne detection.

The NIST test results are again examined as the digitization settings vary in Figs. 13 and 14. In Fig. 13, the gray curves show the results for the individual channels as the detection window size of the ADCs is varied. When the window size is $2.0 \sigma$, no channel can pass all 15 tests because the input signals of the ADCs often exceed the detection windows. As the detection window size increases, the signals exceed the detection windows less often and so more passes are obtained. The interleaved output bits also pass more tests as the detection window size increases, which are shown by the black curve in Fig. 13. The detection window size is then fixed at $4.5 \sigma$ in Fig. 14 as the number of LSBs selected per sample is varied. The output bit rate varies accordingly due to the fixed sampling rate of $10 \mathrm{GHz}$. The gray curves show the results of the individual channels. All 15 tests are passed when up to $5 \mathrm{LSBs}$ are selected per sample in yielding $50 \mathrm{~Gb} / \mathrm{s}$ per channel. The black curve shows the NIST results for the interleaved output bits, which also pass all 15 tests when up to 5 LSBs are selected per sample. The maximum output bit rate 


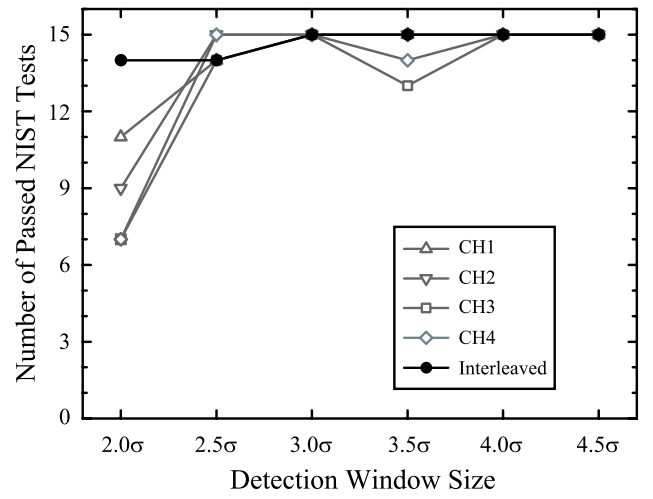

Fig. 13. Number of NIST tests passed versus the detection window size of the ADCs for electrical heterodyne RBG. The NIST tests are conducted on the $50-\mathrm{Gb} / \mathrm{s}$ output bits from $\mathrm{CH} 1, \mathrm{CH} 2, \mathrm{CH} 3$, and $\mathrm{CH} 4$ separately. The sampling rate is $10 \mathrm{GHz}$ with $5 \mathrm{LSBs}$ selected per sample. The tests are also conducted on the interleaved output bits from the four channels with a total output rate of $200 \mathrm{~Gb} / \mathrm{s}$.

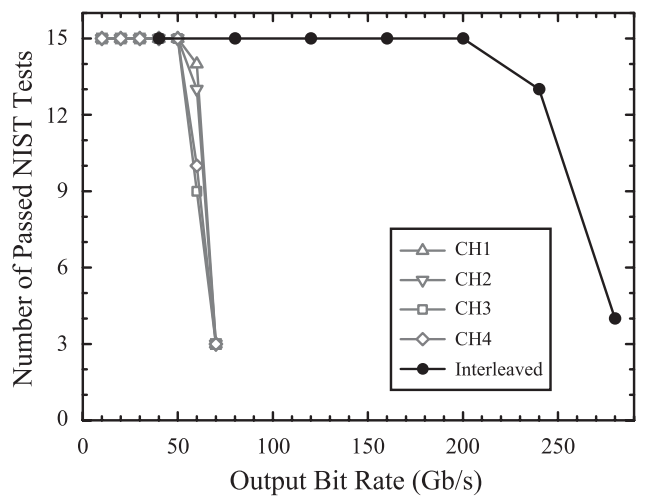

Fig. 14. Number of NIST tests passed versus the output bit rate for electrical heterodyne RBG. The output bit rate is varied by adjusting the number of LSBs selected per sample. The sampling rate is fixed at $10 \mathrm{GHz}$. The detection window size of the ADCs is kept at $4.5 \sigma$. The NIST tests are conducted on $\mathrm{CH} 1, \mathrm{CH} 2, \mathrm{CH} 3, \mathrm{CH} 4$, and their interleaved output bits.

is thus $200 \mathrm{~Gb} / \mathrm{s}$ from the four channels collectively. Without the chaotic emission, the electronic noise of the system in Fig. 1 cannot generate random bits for more than 1 LSB per sample. Hence, the chaotic emission together with heterodyne detection are essential for achieving the high-speed parallel RBG.

\section{Discussions}

Both optical and electrical heterodyne RBG have the advantage of not requiring high-bandwidth ADCs. The chaos spectrum is divided into different portions for effective parallel processing through multiple ADCs. High output rates in the order of $100 \mathrm{GHz}$ are demonstrated using ADCs with frontend bandwidths of only $2.5 \mathrm{GHz}$, which is smaller than the chaos bandwidth of $7.5 \mathrm{GHz}$.

However, the two approaches have their own advantages and disadvantages. For optical heterodyne RBG in Fig. 1(b-i), the electronic bandwidths of the photodetectors and microwave amplifiers can be as small as the front-end bandwidths of the ADCs; although careful polarization control is necessary to optimize the optical interference at FC2. For electrical heterodyne RBG in Fig. 1(b-ii), polarization control is not necessary as no optical interference is involved in the detection of the chaotic emission; although a photodetector and microwave amplifiers with wide bandwidths are needed for efficient utilization of the chaos spectrum.

Every channel in both optical and electrical heterodyne RBG adopts digital processing as shown in Fig. 2. The digital processing involves XOR operations between the original samples and their delayed replicas for reducing the bias of bits [10], [52]. Similar operations between the original samples and the delayed samples were commonly adopted in related works [4], [9], [10], [13], [16]. The choice of the delay time is not critical to the quality of the output bits as long as it is large enough to avoid correlating the original and the delayed samples [10]. Typically, the delay time is in the order of nanoseconds for convenience, which can be realized physically. For instance, a delay time of $4.6 \mathrm{~ns}$ was implemented using a coaxial cable of $1 \mathrm{~m}$ [4]. Nonetheless, both $100-\mathrm{Gb} / \mathrm{s}$ optical heterodyne RBG and $200-\mathrm{Gb} / \mathrm{s}$ electrical heterodyne RBG in Section III are tested using delay times of up to $200 \mathrm{~ns}$. It is observed that the output bits always pass the 15 NIST tests regardless of the value of the delay.

\section{CONClusion}

In conclusion, an optically injected semiconductor laser in chaos is investigated for parallel RBG through heterodyne detection. Optical heterodyne RBG at $100 \mathrm{~Gb} / \mathrm{s}$ using two channels and electrical heterodyne RBG at $200 \mathrm{~Gb} / \mathrm{s}$ using four channels are demonstrated. Each channel employs a low-bandwidth ADC with a front-end supporting only up to $2.5 \mathrm{GHz}$ without boosting the bit rate by postprocessing. The high-speed parallel RBG is enabled by using different portions of the chaos spectrum through heterodyning. While the chaotic laser is subject to $\mathrm{CW}$ optical injection in the experiment, chaotic emissions from other techniques can potentially incorporate the heterodyne approaches for high-speed parallel RBG as well.

\section{REFERENCES}

[1] A. Uchida, K. Amano, M. Inoue, K. Hirano, S. Naito, H. Someya, I. Oowada, T. Kurashige, M. Shiki, S. Yoshimori, K. Yoshimura, and P. Davis, "Fast physical random bit generation with chaotic semiconductor lasers," Nature Photon., vol. 2, no. 12, pp. 728-732, Dec. 2008.

[2] I. Reidler, Y. Aviad, M. Rosenbluh, and I. Kanter, "Ultrahigh-speed random number generation based on a chaotic semiconductor laser," Phys. Rev. Lett., vol. 103, no. 2, pp. 024102-1-024102-4, Jul. 2009.

[3] I. Kanter, Y. Aviad, I. Reidler, E. Cohen, and M. Rosenbluh, "An optical ultrafast random bit generator," Nature Photon., vol. 4, no. 1, pp. 58-61, Jan. 2010.

[4] T. Yamazaki and A. Uchida, "Performance of random number generators using noise-based superluminescent diode and chaos-based semiconductor lasers," IEEE J. Sel. Topics Quantum Electron., vol. 19, no. 4, p. 0600309, Jul./Aug. 2013.

[5] T. Honjo, A. Uchida, K. Amano, K. Hirano, H. Someya, H. Okumura, K. Yoshimura, P. Davis, and Y. Tokura, "Differentialphase-shift quantum key distribution experiment using fast physical random bit generator with chaotic semiconductor lasers," Opt. Exp., vol. 17, no. 11, pp. 9053-9061, May 2009.

[6] I. Kanter, M. Butkovski, Y. Peleg, M. Zigzag, Y. Aviad, I. Reidler, M. Rosenbluh, and W. Kinzel, "Synchronization of random bit generators based on coupled chaotic lasers and application to cryptography," Opt. Exp., vol. 18, no. 17, pp. 18292-18302, Aug. 2010. 
[7] T. Harayama, S. Sunada, K. Yoshimura, P. Davis, K. Tsuzuki, and A. Uchida, "Fast nondeterministic random-bit generation using on-chip chaos lasers," Phys. Rev. A, vol. 83, no. 3, pp. 031803-1-031803-4, Mar. 2011

[8] A. Argyris, S. Deligiannidis, E. Pikasis, A. Bogris, and D. Syvridis, "Implementation of $140 \mathrm{~Gb} / \mathrm{s}$ true random bit generator based on a chaotic photonic integrated circuit," Opt. Exp., vol. 18, no. 18, pp. 18763-18768, Aug. 2010.

[9] J. Z. Zhang, Y. C. Wang, M. Liu, L. G. Xue, P. Li, A. B. Wang, and M. J. Zhang, "A robust random number generator based on differential comparison of chaotic laser signals," Opt. Exp., vol. 20, no. 7, pp. 7496-7506, Mar. 2012.

[10] K. Hirano, T. Yamazaki, S. Morikatsu, H. Okumura, H. Aida, A. Uchida, S. Yoshimori, K. Yoshimura, T. Harayama, and P. Davis, "Fast random bit generation with bandwidth-enhanced chaos in semiconductor lasers," Opt. Exp., vol. 18, no. 6, pp. 5512-5524, Mar. 2010.

[11] P. Li, Y. C. Wang, and J. Z. Zhang, "All-optical fast random number generator," Opt. Exp., vol. 18, no. 19, pp. 20360-20369, Sep. 2010

[12] Y. Y. Zhang, J. Z. Zhang, M. J. Zhang, and Y. C. Wang, "2.87-Gb/s random bit generation based on bandwidth-enhanced chaotic laser," Chin. Opt. Lett., vol. 9, no. 3, pp. 031404-1-031404-3, Mar. 2011.

[13] Y. Akizawa, T. Yamazaki, A. Uchida, T. Harayama, S. Sunada, K. Arai, K. Yoshimura, and P. Davis, "Fast random number generation with bandwidth-enhanced chaotic semiconductor lasers at $8 \times 50 \mathrm{~Gb} / \mathrm{s}$," IEEE Photon. Technol. Lett., vol. 24, no. 12, pp. 1042-1044, Jun. 2012.

[14] R. M. Nguimdo, G. Verschaffelt, J. Danckaert, X. Leijtens, J. Bolk, and G. Van der Sande, "Fast random bits generation based on a single chaotic semiconductor ring laser," Opt. Exp., vol. 20, no. 27, pp. 28603-28613, Dec. 2012

[15] P. Li, Y. C. Wang, A. B. Wang, and B. J. Wang, "Fast and tunable all-optical physical random number generator based on direct quantization of chaotic self-pulsations in two-sectionsemiconductor lasers," IEEE J. Sel. Topics Quantum Electron., vol. 19, no. 4, p. 0600208, Jul./Aug. 2013.

[16] J. G. Wu, X. Tang, Z. M. Wu, G. Q. Xia, and G. Y. Feng, "Parallel generation of $10 \mathrm{Gbits} / \mathrm{s}$ physical random number streams using chaotic semiconductor lasers," Laser Phys., vol. 22, no. 10, pp. 1476-1480, Oct. 2012.

[17] N. Oliver, M. C. Soriano, D. W. Sukow, and I. Fischer, "Dynamics of a semiconductor laser with polarization-rotated feedbackand its utilization for random bit generation," Opt. Lett., vol. 36, no. 23, pp. 4632-4634, Dec. 2011.

[18] X. Z. Li and S. C. Chan, "Random bit generation using an optically injected semiconductor laser in chaos with oversampling," Opt. Lett., vol. 37, no. 11, pp. 2163-2165, Jun. 2012.

[19] V. Demchik, "Pseudo-random number generators for Monte Carlo simulations on ATI graphics processing units," Comput. Phys. Commun., vol. 182 , no. 3, pp. 692-705, Mar. 2011.

[20] J. Chen, J. Zhou, and K. W. Wong, "A modified chaos-based joint compression and encryption scheme," IEEE Trans. Circuits Syst. II, Exp. Briefs, vol. 58, no. 2, pp. 110-114, Feb. 2011.

[21] K. Yoshimura, J. Muramatsu, P. Davis, T. Harayama, H. Okumura, S. Morikatsu, H. Aida, and A. Uchida, "Secure key distribution using correlated randomness in lasers driven by common random light," Phys. Rev. Lett., vol. 108, no. 7, pp. 070602-1-070602-5, Feb. 2012.

[22] J. Muramatsu, K. Yoshimura, and P. Davis, "Information theoretic security based on bounded observability," Lect. Notes Comput. Sci., vol. 5973, pp. 128-139, 2010.

[23] T. Durt, C. Belmonte, L. P. Lamoureux, K. Panajotov, F. Van den Berghe, and H. Thienpont, "Fast quantum-optical random-number generators," Phys. Rev. A, vol. 87, no. 2, pp. 022339-1-022339-10, Feb. 2013.

[24] M. Wahl, M. Leifgen, M. Berlin, T. Rohlicke, H. J. Rahn, and O. Benson, "An ultrafast quantum random number generator with provably bounded output bias based on photon arrival time measurements," Appl. Phys. Lett., vol. 98, no. 17, pp. 171105-1-171105-3, Apr. 2011.

[25] M. A. Wayne and P. G. Kwiat, "Low-bias high-speed quantum random number generator via shaped optical pulses," Opt. Exp., vol. 18, no. 9, pp. 9351-9357, Apr. 2010.

[26] M. Y. Zhu, Y. Liu, Q. F. Yu, and H. Guo, "Random number generation based on polarization mode noise of vertical-cavity surface-emitting lasers," Laser Phys. Lett., vol. 9, no. 11, pp. 775-780, Nov. 2012.

[27] V. N. Chizhevsky, "Symmetrization of single-sided or nonsymmetrical distributions: The way to enhance a generation rate of random bits from a physical source of randomness," Phys. Rev. E, vol. 82, no. 5, pp. 050101-1-050101-4, Nov. 2010.
[28] F. H. Xu, B. Qi, X. F. Ma, H. Xu, H. X. Zheng, and H. K. Lo, "Ultrafast quantum random number generation based on quantum phase fluctuations," Opt. Exp., vol. 20, no. 11, pp. 12366-12377, May 2012.

[29] M. Jofre, M. Curty, F. Steinlechner, G. Anzolin, J. P. Torres, M. W. Mitchell, and V. Pruneri, "True random numbers from amplified quantum vacuum," Opt. Exp., vol. 19, no. 21, pp. 20665-20672, Oct. 2011.

[30] C. Gabriel, C. Wittmann, D. Sych, R. Dong, W. Mauerer, U. L. Andersen, C. Marquardt, and G. Leuchs, "A generator for unique quantum random numbers based on vacuum states," Nature Photon., vol. 4, no. 10, pp. 711-715, Oct. 2010.

[31] T. Symul, S. M. Assad, and P. K. Lam, "Real time demonstration of high bitrate quantum random number generation with coherent laser light," Appl. Phys. Lett., vol. 98, no. 23, pp. 231103-1-231103-3, Jun. 2011.

[32] X. Li, A. B. Cohen, T. E. Murphy, and R. Roy, "Scalable parallel physical random number generator based on a superluminescent LED," Opt. Lett., vol. 36, no. 6, pp. 1020-1022, Mar. 2011.

[33] S. Sunada, T. Harayama, K. Arai, K. Yoshimura, K. Tsuzuki, A. Uchida, and P. Davis, "Random optical pulse generation with bistable semiconductor ring lasers," Opt. Exp., vol. 19, no. 8, pp. 7439-7450, Apr. 2011.

[34] W. Wei, G. D. Xie, A. H. Dang, and H. Guo, "High-speed and bias-free optical random number generator," IEEE Photon. Technol. Lett., vol. 24, no. 6, pp. 437-439, Mar. 2012

[35] A. Argyris, E. Pikasis, S. Deligiannidis, and D. Syvridis, "Sub-Tb/s physical random bit generators based on direct detection of amplified spontaneous emission signals," J. Lightw. Technol., vol. 30, no. 9, pp. 1329-1334, May 2012.

[36] C. R. S. Williams, J. C. Salevan, X. Li, R. Roy, and T. E. Murphy, "Fast physical random number generator using amplified spontaneous emission," Opt. Exp., vol. 18, no. 23, pp. 23584-23597, Nov. 2010.

[37] B. Wetzel, K. J. Blow, S. K. Turitsyn, G. Millot, L. Larger, and J. M. Dudley, "Random walks and random numbers from supercontinuum generation," Opt. Exp., vol. 20, no. 10, pp. 11143-11152, May 2012.

[38] D. Brunner, X. Porte, M. C. Soriano, and I. Fischer, "Real-time frequency dynamics and high-resolution spectra of a semiconductor laser with delayed feedback," Sci. Rep., vol. 2, p. 732, Oct. 2012.

[39] M. Virte, K. Panajotov, H. Thienpont, and M. Sciamanna, "Deterministic polarization chaos from a laser diode," Nature Photon., vol. 7, no. 1, pp. 60-65, Jan. 2013.

[40] T. B. Simpson, J. M. Liu, A. Gavrielides, V. Kovanis, and P. M. Alsing, "Period-doubling route to chaos in a semiconductor laser subject to optical injection," Appl. Phys. Lett., vol. 64, no. 26, pp. 3539-3541, Jun. 1994.

[41] V. Kovanis, A. Gavrielides, T. B. Simpson, and J. M. Liu, "Instabilities and chaos in optically injected semiconductor lasers," Appl. Phys. Lett., vol. 67, no. 19, pp. 2780-2782, Nov. 1995.

[42] V. Annovazzi-Lodi, A. Scire, M. Sorel, and S. Donati, "Dynamic behavior and locking of a semiconductor laser subjected to external injection," IEEE J. Quantum Electron., vol. 34, no. 12, pp. 2350-2357, Dec. 1998.

[43] T. Harayama, S. Sunada, K. Yoshimura, J. Muramatsu, K. Arai, A. Uchida, and P. Davis, "Theory of fast nondeterministic physical random-bit generation with chaotic lasers," Phys. Rev. E, vol. 85, no. 4, pp. 046215-1-046215-9, Apr. 2012.

[44] T. Mikami, K. Kanno, K. Aoyama, A. Uchida, T. Ikeguchi, T. Harayama, S. Sunada, K. Arai, K. Yoshimura, and P. Davis, "Estimation of entropy rate in a fast physical random-bit generator using a chaotic semiconductor laser with intrinsic noise," Phys. Rev. E, vol. 85, no. 1, pp. 016211-1-016211-7, Jan. 2012

[45] S. Sunada, T. Harayama, P. Davis, K. Tsuzuki, K. Arai, K. Yoshimura, and A. Uchida, "Noise amplification by chaotic dynamics in a delayed feedback laser system and its application to nondeterministic random bit generation," Chaos, vol. 22, no. 4, pp. 047513-1-047513-9, Dec. 2012.

[46] L. Zunino, M. C. Soriano, and O. A. Rosso, "Distinguishing chaotic and stochastic dynamics from time series by using a multiscale symbolic approach," Phys. Rev. E, vol. 86, no. 4, pp. 046210-1-046210-10, Oct. 2012.

[47] A. Argyris, D. Syvridis, L. Larger, V. Annovazzi-Lodi, P. Colet, I. Fischer, J. Garcia-Ojalvo, C. R. Mirasso, L. Pesquera, and K. A. Shore, "Chaos-based communications at high bit rates using commercial fibreoptic links," Nature, vol. 438, no. 7066, pp. 343-346, Nov. 2005.

[48] M. C. Soriano, J. Garcia-Ojalvo, C. R. Mirasso, and I. Fischer, "Complex photonics: Dynamics and applications of delay-coupled semiconductors lasers," Rev. Modern Phys., vol. 85, no. 1, pp. 421-470, Jan. 2013 
[49] T. Yamazaki, Y. Akizawa, A. Uchida, K. Yoshimura, K. Arai, and P. Davis, "Fast random number generation with bandwidth-enhanced chaos and post-processing," in Proc. Int. Symp. Nonlinear Theory Appl., Oct. 2012, pp. 142-145.

[50] D. Rontani, A. Locquet, M. Sciamanna, and D. S. Citrin, "Loss of time-delay signature in the chaotic output of a semiconductor laser with optical feedback," Opt. Lett., vol. 32, no. 20, pp. 2960-2962, Oct. 2007.

[51] J. G. Wu, Z. M. Wu, G. Q. Xia, and G. Y. Feng, "Evolution of time delay signature of chaos generated in a mutually delay-coupled semiconductor lasers system," Opt. Exp., vol. 20, no. 2, pp. 1741-1753, Jan. 2012.

[52] K. Hirano, K. Amano, A. Uchida, S. Naito, M. Inoue, S. Yoshimori, K. Yoshimura, and P. Davis, "Characteristics of fast physical random bit generation using chaotic semiconductor lasers," IEEE J. Quantum Electron., vol. 45, no. 11, pp. 1367-1379, Nov. 2009.

[53] X. Z. Li and S. C. Chan, "40-Gbps random bit generation by oversampling chaos from an injected semiconductor laser," Proc. SPIE, vol. 8552, pp. 85520K-1-85520K-7, Nov. 2012.

[54] T. B. Simpson, J. M. Liu, K. F. Huang, and K. Tai, "Nonlinear dynamics induced by external optical injection in semiconductor lasers," Quantum Semiclass. Opt., vol. 9, no. 5, pp. 765-784, Oct. 1997.

[55] S. K. Hwang and J. M. Liu, "Dynamical characteristics of an optically injected semiconductor laser," Opt. Commun., vol. 183, nos. 1-4, pp. 195-205, Sep. 2000.

[56] X. Fu, S. C. Chan, Q. Liu, and K. K. Y. Wong, "Broadband optical chaos for stimulated Brillouin scattering suppression in power over fiber," Appl. Opt., vol. 50, no. 25, pp. E92-E96, Sep. 2011.

[57] S. C. Chan, "Analysis of an optically injected semiconductor laser for microwave generation," IEEE J. Quantum Electron., vol. 46, no. 3, pp. 421-428, Mar. 2010.

[58] A. Rukhin, J. Soto, J. Nechvatal, M. Smid, E. Barker, S. Leigh, M. Levenson, M. Vangel, D. Banks, A. Heckert, J. Dray, and S. Vo, "A statistical test suite for random and pseudorandom number generators for cryptographic applications," NIST Special Publication 800-22 Rev. 1a, Apr. 2010

[59] F. Y. Lin and J. M. Liu, "Nonlinear dynamical characteristics of an optically injected semiconductor laser subject to optoelectronic feedback," Opt. Commun., vol. 221, nos. 1-3, pp. 173-180, Jun. 2003.

[60] F. Y. Lin, Y. K. Chao, and T. C. Wu, "Effective bandwidths of broadband chaotic signals," IEEE J. Quantum Electron., vol. 48, no. 8, pp. 1010-1014, Aug. 2012.
[61] S. S. Li, Q. Liu, and S. C. Chan, "Distributed feedbacks for time-delay signature suppression of chaos generated from a semiconductor laser,' IEEE Photon. J., vol. 4, no. 5, pp. 1930-1935, Oct. 2012.

[62] A. Srinivasan, M. Mascagni, and D. Ceperley, "Testing parallel random number generators," Parallel Comput., vol. 29, no. 1, pp. 69-94, Jan. 2003.

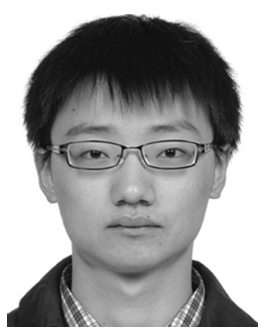

Xiao-Zhou $\mathbf{L i}$ received the B.Eng. degree in communication engineering from the Harbin Institute of Technology, Harbin, China, in 2011. He is now pursuing the M.Phil. degree in electronic engineering with the City University of Hong Kong, Hong Kong, China. His current research interests include random bit generation, optical chaos, and microwave photonics.

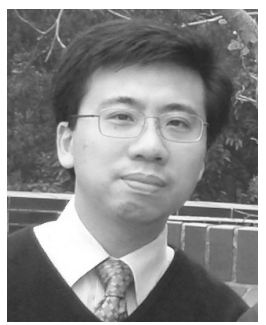

Sze-Chun Chan received the B.Eng. degree in electrical and electronic engineering from the University of Hong Kong, Hong Kong, China, in 2001, and the M.S. and Ph.D. degrees in electrical engineering from the University of California at Los Angeles, Los Angeles, CA, USA, in 2004 and 2007, respectively. He is currently an Associate Professor with the Department of Electronic Engineering and the State Key Laboratory of Millimeter Waves at the City University of Hong Kong, Hong Kong, China. He received the Dr. Bor-Uei Chen Scholarship of the Photonics Society of Chinese-Americans in 2007 and the Departmental Outstanding Teacher Awards from 2009 to 2012. His research interests include nonlinear dynamics of semiconductor lasers, optical chaos generation, radioover-fiber, and photonic microwave generation. 